Title: Thalamic Directional Deep Brain Stimulation for Tremor: Spend Less, Get More

Authors: P Rebelo, RN ${ }^{1,3}$; AL Green, FRCS, PhD ${ }^{1,3}$; T Aziz, FRCS, PhD ${ }^{1,3}$; A Kent, $\mathrm{PhD}^{2}$; D Schafer, $\mathrm{PhD}^{2}$; L Venkatesan, $\mathrm{PhD}^{2}$; B Cheeran, MRCP, $\mathrm{PhD}^{3,4}$

1. Nuffield Department of Surgery, University of Oxford, Oxford, UK

2. R\&D, Abbott, Minneapolis, U.S.A.

3. John Radcliffe Hospital, Oxford, UK.

4. Molecular and Clinical Sciences Research Institute, St George's University of London, London, UK.

Corresponding Author: (Present address) Binith Cheeran, Hon Senior Clinical Fellow, Molecular and Clinical Sciences Research Institute, St George's University of London, London, UK. Email: bcheeran@ @gul.ac.uk Collaborators:

Oxford Clinical Group (B Forrow ${ }^{4}$, C Fletcher $^{4}$, M Bogdanovic ${ }^{4}$, JF Fitzgerald $^{1,4}$, Bilal Mirza $\left.{ }^{1,4}\right)$, E Pereira ${ }^{1,3}$

Running title: Directional Deep Brain Stimulation for Tremor Word Count: words (excluding Abstract and Figure Legends) Number of Figures: 6 Supplementary online methods: Mathematica code for therapy impedance estimation, Template of D-DBS Graphical Programming record, VTA calculation methodology, Lead Position data. 


\title{
Thalamic Directional Deep Brain Stimulation for Tremor: Spend Less, Get More
}

\author{
Abstract: \\ Background: Directional Deep Brain Stimulation (D-DBS) allows axially asymmetric \\ electrical field shaping, away from structures causing side-effects. However, concerns \\ regarding the impact on device lifespan and complexity of the monopolar survey has \\ contributed to sparing use of these features.
}

Objective: To investigate whether chronically implanted D-DBS systems can improve the therapeutic window, without a negative impact on device lifespan, in thalamic deep brain stimulation (DBS).

Methods: We evaluated stable outcomes of initial programming sessions (4-6 weeks postimplantation) retrospectively in 8 patients with drug resistant disabling tremor syndromes. We assessed the impact of directional stimulation on therapeutic window (TW), therapeutic current strength (TCS), tremor scores, disability scores and total electrical energy delivered. Finally, we performed volume of tissue activation (VTA) modelling, based on the average values reported in this study.

Results: We report significant gains in TW (91\%) and reductions in TCS (31\%) with stimulation in the best direction compared to best omnidirectional stimulation alternative. Significant improvements in tremor and ADL scores were sustained at 6 months. There was no increase in averaged IPG power consumption (there is a $6 \%$ reduction over the omnidirectional-only alternative). Illustrative VTA modelling shows that D-DBS achieves $85 \%$ of the total activation volume at just $69 \%$ of the stimulation amplitude of nondirectional configuration.

Conclusions: D-DBS can improve the therapeutic window over non-directional DBS, leading to significant reduction in disability that may sustained post implantation without additional reprogramming visits. Device lifespan was not negatively impacted in this study due to the programming approach used. 


\section{Introduction:}

Directional Deep Brain Stimulation (D-DBS) systems are a recent innovation, enabling 'steering' of the electric field away from structures causing side-effects, in order to improve the therapeutic window (TW) [1,2]. While standard DBS leads have 4 cylindrical shell electrodes, commercialised D-DBS lead designs have replaced the middle two electrodes with tri-trodes ( 3 individual electrode segments). Each segmented electrode within a tri-trode addresses less than 1/3rd of the circumference of the lead, enabling a volume of tissue activation (VTA) that is asymmetric around the long axis of the lead.

D-DBS systems can be programmed omnidirectionally - producing VTAs that are largely symmetrical around the long axis of the DBS lead (like standard DBS)- by activating all three segmented electrodes in a tri-trode concurrently. One approach to programming D-DBS systems has been to explore directional stimulation only when necessary- the 1680-fold increase in electrode permutations has an impact on clinical resources and patient discomfort during initial monopolar survey. Concerns about reduced lifespan of the Implantable Pulse Generator (IPG), due to stimulation through higher impedance (smaller surface area) segmented electrodes, also contribute to sparing use of directional stimulation features.

However, intra-operative pilot studies have suggested that D-DBS systems may not just improve TW but also lower the strength of stimulation required to produce desired clinical benefit (Therapeutic Current Strength (TCS)) by enabling more efficient stimulation of the target structure[3].

We introduced a structured monopolar survey protocol alongside a graphical documentation system, to simplify and standardise the D-DBS monopolar survey at our centre (see Fig 1). The protocol utilises directional stimulation features to not only avoid side-effects, but also extend IPG life. We applied this system to study the utility of D-DBS in ventral intermediate (VIM) thalamic nucleus for drug-refractory tremor syndromes. Moreover, we wished to study the effects of directional stimulation on predicted IPG lifespan. 


\section{Materials and Methods:}

\section{Experimental design:}

The study adheres to the principles outlined in the Declaration of Helsinki.

Data from all patients implanted in the ventral intermediate (VIM) thalamic nucleus for drugrefractory tremor syndromes, within 6 months of the introduction of the Infinity D-DBS system (Abbott, Minneapolis), at the John Radcliffe Hospital, Oxford, were analysed retrospectively. This D-DBS system offers a $1.5 \mathrm{~mm}$ inter-electrode spacing directional lead preferred for thalamic DBS. A total of 8 patients ( 7 males, 1 female, age 60-72, were selected to undergo VIM DBS (between April 2016 to October 2016) for drug refractory disabling tremor syndromes. This includes Tremor-Predominant Parkinson's Disease (not suited for STN DBS due to age, and paucity of other symptoms or excellent response of nontremor symptoms to levodopa), Dystonic Tremor or Tremor with Dystonia elsewhere (with non-symptomatic or minor dystonic features that did not require treatment) or Essential Tremor (see Table 1 for demographic details).

\section{Targeting:}

Imaging based targeting of VIM was done using a 1.5T MRI scan, as previously published by our Neurosurgical service[4] (For the Left VIM x axis mean was $-14.51 \mathrm{~mm}$, SD 2.12mm; y axis mean was $-4.21 \mathrm{~mm}$ SD $0.64 \mathrm{~mm}$; $\mathrm{z}$ axis mean was $0.11 \mathrm{~mm}$ SD $0.40 \mathrm{~mm}$ from AC-PC line. For the right VIM $x$ axis mean was $13.85 \mathrm{~mm}$, SD $1.91 \mathrm{~mm}$; $y$ axis mean was $-4.20 \mathrm{~mm}$ $\mathrm{SD} 0.85 \mathrm{~mm} ; \mathrm{z}$ axis mean was $0.17 \mathrm{~mm} \mathrm{SD} 0.41 \mathrm{~mm}$ from AC-PC). The surgery was conducted with the patient awake, enabling intra-operative clinical assessment of lead placement. The lead was placed such that the radio-opaque marker towards the distal end of the lead (corresponding to contact 2A) faced anteriorly. Lead placement was confirmed with a postoperative CT scan fused to pre-operative MRI. (Left VIM lead location mean for $\mathrm{x}$ axis was $13.64 \mathrm{~mm}$ SD $2.10 \mathrm{~mm}$, z axis $-4.68 \mathrm{~mm}$ SD 1.33 , y axis $0.30 \mathrm{~mm}$ SD $0.51 \mathrm{~mm}$, Right VIM lead location mean for $\mathrm{x}$ axis $13.77 \mathrm{~mm}$ SD $2.69 \mathrm{~mm}$; $\mathrm{y}$ axis $-4.99 \mathrm{~mm}$ SD $1.31 \mathrm{~mm}$; $\mathrm{z}$ axis $0.17 \mathrm{~mm}$ SD $0.42 \mathrm{~mm}$ ). Details are provided in supplementary data.

Euclidean distance from intended stereotactic target to actual implanted lead position was measured in $\mathrm{X}$ and $\mathrm{Y}$ axis, at the level of the stereotactic target. Orientation of DBS segments was recorded by A-P and lateral cranial X-rays. As reported by other groups, determining 
orientation of segmented to the degree of accuracy required to reliably inform or analyse programming was not possible using cranial X-rays. Despite efforts to place the lead with segment 2A facing anteriorly, torque on the lead during fixation (with a dog-bone collar) often led to some deviation from this intended orientation. Details of marker orientation is provided in supplementary data.

\section{Programming protocol key steps:}

Patients were evaluated postoperatively (Mean=45 Days, SD=7 Days) as part of routine clinical care. Some patients were assessed over more than one session within the first 8 weeks to obtain optimum stimulation parameters. In all cases, the stable outcome of initial programming sessions (in all cases less than 8 weeks post implantation) was analysed. TW and TCS at the Best Segmented Level (BSL) (the segmented contact level with the widest therapeutic window in omnidirectional stimulation) recorded in the graphical documentation scheme (Fig 1) was evaluated independently by a second experienced DBS programmer (PR, $\mathrm{BC})$ prior to inclusion.

Screening of contacts was not informed by the lead location or orientation determined on post-operative imaging. Rather, each contact was assessed clinically, without restricting screening to contacts predicted by post-operative imaging. Pulse width (90 microseconds) and frequency $(130 \mathrm{~Hz})$ was fixed during the monopolar review, and only amplitude was varied to screen each contact. TW was defined as difference in current strengths at which tremor suppression was obtained and first sustained side-effect occurred. Initially, nondirectional stimulation was assessed for each non-segmented electrode and tri-trode activation. Then, TW for directional stimulation through each individual segmented electrode was assessed. While screening individual segmented electrodes, step size for increments in current strength (amplitude) was restricted to 10\% of TCS recorded for omnidirectional stimulation at that level, to account for the higher energy densities and larger VTAs achieved by stimulation through a smaller contact. Typically, this meant that segmented contacts were examined in $0.2 \mathrm{~mA}$ increments. Without this crucial step, segmented electrodes are not examined at a similar resolution to non-segmented electrodes. Testing of co-activation of pairs of segmented electrodes was advised adjacent if the TW was similar in adjacent segmented contacts. 
Directional stimulation was selected if a sustained side-effect (even mild sustained sideeffects) could be prevented. Although the smaller segmented electrodes have higher impedances (1.8-2 times that of cylindrical electrodes or tri-trodes typically), power consumption can still be lower if lower stimulation parameters are achieved. If TCS at any segmented contact was $30 \%$ lower than omnidirectional stimulation at that level, exploration of potential benefit of directional stimulation on IPG lifespan was advised, based on the trade-off point between lower amplitude and typical higher impedance.

\section{VTA modelling:}

A two-stage computational model (Sim4Life v3.2) was used to calculate VTA resulting from stimulation with the Infinity D-DBS lead. The first stage involved using a finite element analysis (FEA) model to calculate electrical potentials generated in brain tissue during DBS (detailed in supplementary data). The electrical potentials from the FEA model were interpolated along each axon, and delivered as extracellular stimulation to determine which axons were activated for each stimulation set. VTAs were calculated for the non-directional and directional configurations using methods adapted from Butson and colleagues[5]. Briefly, the second difference of the extracellular potentials $\left(\Delta^{2} V_{e} / \Delta x^{2}\right)$ was calculated at the nodes of Ranvier along all axons. The threshold predictor value was then determined as the value of $\Delta^{2} V_{e} / \Delta x^{2}$ at the node of Ranvier where action potential initiation occurred that was farthest away from the center of contact 2. Finally, the VTA was calculated all the volume encompassing all nodes of Ranvier in which the value of $\Delta^{2} V_{\mathrm{e}} / \Delta \mathrm{x}^{2}$ exceeded the threshold predictor value (excluding those nodes that intersected with the DBS electrode). The extent of directionality was calculated as the percentage of VTA volume on the side of the lead with segment $2 \mathrm{~A}$.

\section{Energy use estimation:}

Predicted IPG lifespan (calculated by the Longevity Calculator clinician programmer with minimal of 8 days of program as per manufacture recommendations), was recorded at the same visit. Additionally, Total Electrical Energy Delivered (TEED) scores were calculated for all leads, both for the actual stimulation parameters determined by the programming clinician (directional or omnidirectional), and the best omnidirectional stimulation alternative 
for those leads programmed directionally. To calculate the TEED score of the best omnidirectional option, the impedance was estimated using a simplified resistance model (see supplementary data). Change in TEED from best omnidirectional stimulation alternative was calculated using the formula [1- (TEED Best Omnidirectional Alternative/ TEED Actual Programmed Parameter)].

\section{Statistical Analysis:}

2-tailed paired T-tests were used to improvements in Activities of Daily Living (ADL) scores after surgery, effects of directional and omnidirectional DBS on TCS and TW. A 2-tailed Ttest was used to assess the effect of lead implant error on uptake of D-DBS.

\section{Results:}

All 8 patients received bilateral implants, but 1 lead was not assessed due to persistent stun effect in the less affected limb for that patient (final $n=15$ leads, 8 patients). $7 / 8$ of patients were deemed to have gained additional benefit from directional stimulation on at least one side. 9/15 leads utilised directional stimulation (6 to avoid side-effects, 3 to improve IPG lifespan). Lead implant error did not affect uptake of D-DBS features in this small sample and grand mean of lead implant error was $1 \mathrm{~mm}$ in $\mathrm{X}$ (medio-lateral) and $\mathrm{Y}$ (antero-posterior) axis at the level of intended target $(\mathrm{z}=0)$ (Fig 2). On average, ADL scores improved by $56 \%$ at 3 months, and this improvement was maintained unchanged at 6 months (Bain-Findley Tremor scale ADL subscale) (Figure 3, only scores at 6 month time-point shown) (2-tailed paired T-test, $\mathrm{p}=0.001$ )[6]. Average tremor scores were improved by $86 \%$ at 6 months compared to pre-operative scores (Fig 3)(2-tailed paired T-test, $\mathrm{p}<0.001$ ). Notably, stimulation parameters determined by week 8 were unchanged at week 24 . At BSL, directional stimulation significantly improved TW over non-directional stimulation (2-tailed paired T-test, $\mathrm{p}=0.0004$ ) (Fig 4). At BSL, directional stimulation achieved desired clinical effects at significantly lower TCS compared to non-directional stimulation (2-tailed paired Ttest, $\mathrm{p}=0.0001$ ) (Fig 4). VTA modelling was done to better understand these results (Fig 5). VTA modelling using average TA for omnidirectional and directional stimulation in our study illustrates that D-DBS can produce a VTA that is roughly $1 / 3^{\text {rd }}$ larger in the desired directional, at $1 / 3^{\text {rd }}$ less stimulation amplitude compared to omnidirectional stimulation (Fig 
5). Average predicted IPG lifespan (by the Longevity Calculator function) for this cohort was 56.6 months. Average TEED for actually programmed parameters was $6 \%$ lower than if all leads were to be programmed omnidirectionally (Fig 6A).

\section{Discussion:}

We report, for the first time, the utility of a D-DBS system in Thalamic DBS for the treatment of drug-refractory tremor syndromes. Statistically significant gains in TW $(91 \%$ wider) and TCS (31\% lower) occurred with stimulation in the best direction compared with omnidirectional stimulation. Statistically significant improvements in ADL and tremor scores were maintained at 6 months, without the need for parameter adjustment between initial programming sessions and the 6-month follow-up time point. The stability of stimulation settings between week 8 and 24 is notable. Continued follow-up of this cohort shows that only a single patient, with a significant lead placement error limiting therapy and impact on tremor suppression, is due to be reviewed for re-programming at 8 months post implant. This suggests that any additional time spent screening individual segmented contacts may be recouped as disease progression demands increased TCS in subsequent visits. Although there is no formal comparison was conducted, in our experience, a reductive approach to screening D-DBS electrodes has meant that initial monopolar survey and documentation requires no more than approximately $25 \%$ more time than the standard non-directional monopolar review. This study is, therefore, the first D-DBS study to report stable outcomes of initial programming sessions and clinical outcomes at 6 months post implant of directional DBS systems. Prior studies of D-DBS have focussed on STN DBS for Parkinson's Disease, as millimetric error in lead placement can lead to loss of therapy benefit or produce therapylimiting side effects with this target. Our study suggests that D-DBS may be as relevant in thalamic DBS, if not more so, as minor side-effects (particularly dysarthria) are relatively common with bilateral thalamic DBS for tremor.

There is no apparent effect on lead placement error on mode of programming, perhaps in part because directional stimulation is also selected for IPG lifespan. 
The limited evidence for D-DBS systems has come from VTA modelling studies, safety studies in animals and acute intraoperative proof-of-concept studies in humans[7]. Pollo et al. and Contarino et al. published acute intraoperative proof-of-concept studies, where patients (mostly with Parkinson's disease undergoing STN DBS) underwent double-blinded intraoperative evaluation of omnidirectional and directional stimulation with investigational directional electrode prototypes, that were then replaced with a standard non-directional electrode[3,8]. Pollo et al reported significant improvements in TW (41.3\% wider) and in TCS (43\% lower) with stimulation in the best direction than with omnidirectional stimulation. Contarino et al. reported improvements in side-effect thresholds, but only two (of 8) patients showed an improved TW. Only one case-series has reported the post-operative evaluation of chronically implanted directional DBS. Steigerwald et al. meticulously evaluated omnidirectional and directional stimulation (not blinded), with Boston Scientific Cartesia $^{\mathrm{TM}}$ lead/ Vercise ${ }^{\mathrm{TM}}$ IPG, during exploratory programming in the immediate postoperative period (just 4 to 9 days' post-surgery)[9]. This study did not show a statistically significant gain in therapeutic window between directional and omnidirectional stimulation, likely due to persistent microlesional effects reducing therapy demands.

In this study, we have also attempted to address the impact of D-DBS on IPG lifespan. There is no negative impact (in fact there is a small gain) on IPG lifespan despite the frequent use of D-DBS features across this cohort. With program impedance for a single segmented contact being around 1.8-2.2 times that of full tri-trode activation for Infinity leads, IPG life can be extended if directional stimulation reduces TCS by at least $26 \%$. Indeed, this study found that reduction in TCS for directional stimulation over full tri-trode activation at BSL was $31 \%$, therefore suggesting that the IPG lifespan may be extended with directional stimulation.

The VTA modelling illustrates that D-DBS may not only improve symptom control by providing a stimulation field customized to the lead location and concentrated on where it has most impact, but can do so efficiently by producing equivalent VTAs at lower TCS. This is not intuitive and poorly recognised- that stimulation through a smaller $\left(1 / 3^{\text {rd }}\right.$ surface area) segmented electrode produces a VTA that is substantially larger than that produced through full tri-trode activation at equivalent stimulation settings. Higher current density at the electrode-tissue interface and the 'edge' effect are two factors that may underpin this[7,10]. 
The value of a full directional survey is confirmed by the TEED comparison. If leads were only programmed directionally when side-effects were encountered, there would have been an $18 \%$ increase from the average TEED for actually programmed parameters in this study (Fig 6B). This would equate to as much as a year of IPG lifespan lost if directional stimulation was only used when side-effects are encountered in this cohort.

The limitations of this study include the small size of this cohort, the retrospective nature of the study, the lack of blinding and short duration of follow-up (6 months post implant). Larger prospective studies are required to confirm these preliminary findings.

D-DBS systems are a welcome innovation, after a decade of minimal technological advance in DBS therapy. As with any new technology, there is a lag between the introduction of new features, and establishing how to optimise benefits and prevent misuse. Future developments such as expert systems to aid optimal electrode selection (for example detecting when IPG life can be extended) may be key to optimising our use of this promising new technology.

\section{Acknowledgements:}

$\mathrm{BC}$ was funded by the Oxford Biomedical Research Centre

\section{Author contributions:}

BC conceived the study. PR and BC collected and analysed data. PR, supervised by AG and TA, audited implant accuracy data. VTA modelling was conducted by AK with contributions from LV and BC. Impedance estimates were calculated by DS and LV. BC, with input from all authors, authored the manuscript.

\section{Clinical Collaborators:}

B Forrow, C Fletcher, M Bogdanovic, JF Fitzgerald and B Mirza were involved in the routine clinical care and evaluation of DBS patients. The programming protocol/ documentation system for directional systems devised by BC was implemented for all patients with D-DBS systems by $\mathrm{BC}, \mathrm{BF}, \mathrm{CF}$ and $\mathrm{PR}$ prior to and during this study, as part of routine care. $\mathrm{E}$ Pereira provided $\mathrm{BC}$ with software and material support during data analysis.

\section{Author Conflict of Interest Statement:}


BC, PR and AG hold consultancy contracts with Boston Scientific and Abbott, manufacturers of Directional DBS devices. TA has served as a consultant for Medtronic Neuromodulation, a manufacturer of DBS devices and investigational Directional DBS device. LV, AK and DS are current employees of Abbott. 


\section{References:}

[1] Kühn AA, Volkmann J. Innovations in deep brain stimulation methodology. Mov Disord 2017;32:11-9. doi:10.1002/mds.26703.

[2] Hariz M. Deep brain stimulation: new techniques. Parkinsonism Relat Disord 2014;20 Suppl 1:S192-6. doi:10.1016/S1353-8020(13)70045-2.

[3] Pollo C, Kaelin-Lang A, Oertel MF, Stieglitz L, Taub E, Fuhr P, et al. Directional deep brain stimulation: an intraoperative double-blind pilot study. Brain 2014;137:2015-26. doi:10.1093/brain/awu102.

[4] Bittar RG, Burn SC, Bain PG, Owen SL, Joint C, Shlugman D, et al. Deep brain stimulation for movement disorders and pain. vol. 12. Elsevier; 2005. doi:10.1016/j.jocn.2004.09.001.

[5] Butson CR, McIntyre CC. Current steering to control the volume of tissue activated during deep brain stimulation. Brain Stimul 2008;1:7-15. doi:10.1016/j.brs.2007.08.004.

[6] Bain PG, Findley LJ, Atchison P, Behari M, Vidailhet M, Gresty M, et al. Assessing tremor severity. J Neurol Neurosurg Psychiatr 1993;56:868-73.

[7] Butson CR, Venkatesan L. Comparison of neural activation between standard cylindrical and novel segmented electrode designs. Mov Disord 2014;29.

[8] Contarino MF, Bour LJ, Verhagen R, Lourens MAJ, de Bie RMA, van den Munckhof P, et al. Directional steering: A novel approach to deep brain stimulation. Neurology 2014;83:1163-9. doi:10.1212/WNL.0000000000000823.

[9] Steigerwald F, Müller L, Johannes S, Matthies C, Volkmann J. Directional deep brain stimulation of the subthalamic nucleus: A pilot study using a novel neurostimulation device. Mov Disord 2016;31:1240-3. doi:10.1002/mds.26669.

[10] Wei XF, Grill WM. Current density distributions, field distributions and impedance analysis of segmented deep brain stimulation electrodes. J Neural Eng 2005;2:139_ 47. doi:10.1088/1741-2560/2/4/010. 


\section{Figure Legends:}

Fig 1. Documentation and Programming scheme.

A) Marker orientation- if known, radio-opaque marker used to determine the approximate orientation of segmented contacts. Not recorded in this study.

B) Fixed survey parameters (suggested 130hz, and either 60 (STN) or 90 microseconds (Thalamic and GPi)).

C) Additional notes- Qualitative and quantitative information about side-effects and partial benefit.

D) The contact being tested is recorded in the solid grey circle. The grid in this example is scaled at $0.2 \mathrm{~mA}$ per small square (for STN or Thalamic DBS). The dotted circles are labelled to indicate the scale and provide a visual guide to record non-directional stimulation outcomes.

E) Stimulation amplitudes at which desired therapeutic outcome (green circle) and first sustained side-effect occurs (red circle) are recorded as concentric circles for omnidirectional stimulation. The gap between is a visual guide to the therapeutic window at that electrode. Partial benefit and transient side-effects are recorded as dashed green and red circles respectively.

F) The panels for segmented electrodes have a distinctive 3 spoke design. Each thick grey spoke (radial line) is at 120 degrees from the other and represents the direction of stimulation for each segmented electrode. Thin grey spokes in between thicker grey spokes are used to record the effect of co-activation of adjacent segmented electrodes if required. Initially, all three segmented contacts (the 'tri-trode') are activated to produce non-directional stimulation. Then, each segmented contact is tested individually to determine the stimulation amplitudes at which good therapeutic benefit and sustained side-effect occurs. These points are joined up as two polygons (triangles if only the three principal axes are tested in initial screening)- one for good therapeutic benefit (green triangle) and another for sustained side-effect (red triangle). This makes the results of directional surveys visually distinctive from nondirectional stimulation. Recording the results of the monopolar survey in this way enables the programmer to make a quick, intuitive judgment on therapeutic windows, to decide between directional Vs non-directional stimulation and contact selection for therapy. 
Fig 2. Implant accuracy versus uptake of directional programming features.

Mean error across cohort is $1 \mathrm{~mm}$. No statistically significant difference noted in uptake of directional stimulation features with implant accuracy. Accuracy was measured as the difference between intended lead location (on pre-operative planning MRI scan) and final lead position (lead artefact on post-operative CT fused with pre-operative planning MRI).

Fig 3. Tremor and Disability outcomes at 6 months.

Tremor scores were collected pre-operatively and at 6 months. Disability scores were collected pre-operatively, then at three and six months. Disability scores were identical at 3 and 6 months, with no requirement for parameter adjustment or re-programming.

Fig 4. Therapeutic Window (TW) and Therapeutic Current Strength (TCS) gains.

At best segmented level (BSL), directional stimulation (Mean TW 1.86mA, SEM 0.24) significantly improved TW over non-directional stimulation (Mean TW 0.97mA, SEM 0.24 (Fig 2A). At BSL, directional stimulation achieved desired clinical effects at significantly lower TCS (Mean TCS $1.51 \mathrm{~mA}$, SEM 0.18) compared to non-directional stimulation (Mean TCS 2.19 mA, SEM 0.19) (Fig 2B). Therefore, if TW is expressed as a \% of TCS, nondirectional stimulation allows for a $44 \%$ increase in TCS, while directional stimulation allows for a $123 \%$ increase from initial TCS.

Fig 5. VTA modelling provides insight into effects of Directional DBS

VTA modelling at the average TCS for D-DBS through a single segmented contact Vs omnidirectional stimulation through the tri-trode (2ABC) illustrates that the directional configuration achieves $85 \%$ of the total VTA volume at just $69 \%$ of the stimulation amplitude of non-directional configuration. Moreover, VTA volume in the desired direction (side with electrode $2 \mathrm{~A}$ ) is $36 \%$ larger in directional over non-directional configuration.

Fig 6. Effect of Directional Stimulation on Energy Use TEED Score changes by individual lead (left-hand panel) and by programming approach (right-hand panel). Change in TEED from best omnidirectional stimulation alternative is shown for each individual lead (see text for formula). When omnidirectional stimulation is the actual programmed mode of stimulation (Leads 3,5,7,8,12,14), there is no change in 
TEED recorded. Averaged across all leads, TEED for programmed parameters (directional or omnidirectional) is $6 \%$ less compared to that estimated for all leads programmed as best omnidirectional alternative in this cohort. However, if directional programming was only explored or utilised when side-effects were encountered, average TEED for this cohort would have been $12 \%$ greater than omnidirectional stimulation alone. The difference between the two approaches to D-DBS programming is $18 \%$. 